\title{
Natural products as a means of overcoming cisplatin chemoresistance in bladder cancer
}

\author{
Ganeshkumar Rajendran, John A. Taylor III, Benjamin L. Woolbright \\ Department of Urology, University of Kansas Medical Center, Kansas City, KS 66160, USA.
}

Correspondence to: Prof. Benjamin L. Woolbright, Department of Urology, University of Kansas Medical Center, 3901 Rainbow Blvd, MS 1018, Kansas City, KS 66160, USA. E-mail: bwoolbright@kumc.edu

\begin{abstract}
How to cite this article: Rajendran G, Taylor III JA, Woolbright BL. Natural products as a means of overcoming cisplatin chemoresistance in bladder cancer. Cancer Drug Resist 2021;4:69-84. http://dx.doi.org/10.20517/cdr.2020.69
\end{abstract}

Received: 21 Aug 2020 First Decision: 16 Oct 2020 Revised: 5 Nov 2020 Accepted: 12 Nov 2020 Available online: 19 Mar 2021

Academic Editor: Godefridus J. Peters Copy Editor: Cai-Hong Wang Production Editor: Jing Yu

\begin{abstract}
Cisplatin remains an integral part of the treatment for muscle invasive bladder cancer. A large number of patients do not respond to cisplatin-based chemotherapy and efficacious salvage regimens are limited. Immunotherapy has offered a second line of treatment; however, only approximately $20 \%$ of patients respond, and molecular subtyping of tumors indicates there may be significant overlap in those patients that respond to cisplatin and those patients that respond to immunotherapy. As such, restoring sensitivity to cisplatin remains a major hurdle to improving patient care. One potential source of compounds for enhancing cisplatin is naturally derived bioactive products such as phytochemicals, flavonoids and others. These compounds can activate a diverse array of different pathways, many of which can directly promote or inhibit cisplatin sensitivity. The purpose of this review is to understand current drug development in the area of natural products and to assess how these compounds may enhance cisplatin treatment in bladder cancer patients.
\end{abstract}

Keywords: Cisplatin, bladder cancer, natural products, apoptosis

\section{INTRODUCTION}

Bladder cancer $(\mathrm{BCa})$ is a common solid tumor with high rates of morbidity and mortality, especially in patients with advanced disease. Tumor stage is predicated on invasion into the bladder musculature and is linked to both treatments and patient outcomes ${ }^{[1,2]}$. Non-muscle invasive disease is treated with resection of the tumor and is typically followed by intravesical treatment with Bacillus-Calmette-Guerin (BCG)

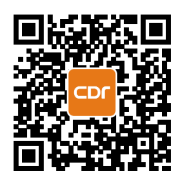


immunotherapy ${ }^{[1]}$. In contrast, muscle invasive disease is typically treated with radical cystectomy ${ }^{[2]}$. In addition, either adjuvant (after surgery) or neoadjuvant (before surgery) cisplatin-based chemotherapy is typically given to patients to reduce tumor burden and to treat potential metastatic disease $\mathrm{e}^{[1,3-6]}$. A complete response to chemotherapy is associated with improved outcomes ${ }^{[3,7]}$. Unfortunately, up to $50 \%$ of patients are resistant to cisplatin-based chemotherapy or develop resistance over time ${ }^{[8]}$. Resistance is further problematic as cisplatin has a number of known toxicities, including cardiotoxicity, ototoxicity, and particularly nephrotoxicity that preclude usage ${ }^{[9,10]}$. This is extremely problematic in a patient population composed largely of elderly patients with high incidences of smoking as well as other co-morbidities. Treating patients that are unlikely to receive a benefit is thus inadvisable and simply increasing dosages in patients that are not initial responders is not safe due to toxicity. As such, advancing cisplatin-based chemotherapy remains a major avenue for improving patient outcomes in patients with muscle-invasive disease.

Natural products derived from plant and animal sources have long been a source of biologically active compounds. Natural product derived compounds such as the taxols, rapamycin, digoxin and more have successfully been translated to the clinic for various diseases; whereas a multitude of other compounds, or extracts from plant sources, remain under investigation for their noted biological activity. Many of these compounds have been reported to enhance the efficacy of cisplatin or block the toxicity in other organs ${ }^{[11,12]}$. Developing these compounds as a means for improving cisplatin therapy offers potential to offer cisplatin to a wider range of patients and improve patient response.

The purpose of this review is to understand the mechanisms that dictate the ability of cisplatin to block proliferation and enhance apoptosis in cancer cells and the mechanisms that result in cisplatin resistance. We will further discuss how compounds derived from natural products can potentially abrogate cisplatin resistance and improve therapeutic response.

\section{CISPLATIN BASED CHEMOTHERAPY IN BLADDER CANCER}

Current chemotherapy regimens used for $\mathrm{BCa}$ include either MVAC (methotrexate, vinblastine, adriamycin, cisplatin) or GC (gemcitabine/cisplatin) $)^{[3,4,7]}$. Both are largely dependent upon response to cisplatin more so than the other compounds. Understanding the molecular mechanisms that determine cisplatin efficacy and predicting which patients will respond to cisplatin treatment remains a major topic of interest in BCa treatment.

\section{Molecular mechanisms}

We will focus on the primary understood mechanisms of cisplatin efficacy and resistance, and how they relate to cancer treatment in the bladder ${ }^{[13,14]}$. Cisplatin uptake is actively mediated by the copper receptor CTR $1^{[15,16]}$. Cellular uptake is required for cisplatin efficacy, as it is largely inactive until it reaches the cytosol where it is biologically activated via aquation ${ }^{[17,18]}$. The active form of cisplatin can adduct or otherwise interact and bind with proteins, DNA, and cellular antioxidants including glutathione (GSH) or free cysteines on proteins such as metalliothionein. Cisplatin adducts are the major source of cellular damage caused by cisplatin, especially cisplatin-DNA adducts formed on purines, particularly guanine ${ }^{[18,19]}$. The formation of DNA adducts also leads to multiple DNA damage response pathways resulting in the activation of ataxia telangiectasia and Rad3-related protein (ATR)/ATM serine/threonine kinase (ATM) and checkpoint kinase 1 (Chk1) and checkpoint kinase $2(\mathrm{Chk} 2)^{[20]}$. ATR/ATM/Chk1/Chk2 activation initiates pro-apoptotic signaling pathways mediated by 553 that converge on the mitochondria, although p53 independent cell death has also been noted, consistent with the fact that cisplatin is used in both p53 WT and p53 mutant tumors ${ }^{[21,22]}$. Cisplatin is often more effective in p53 mutant cells further highlighting the dependency of cisplatin on DNA damage to induce cytotoxicity ${ }^{[9,21]}$. P53 is known to mediate the effects of chemotherapeutics differently between tissues and thus fully understanding how p53, commonly mutated 


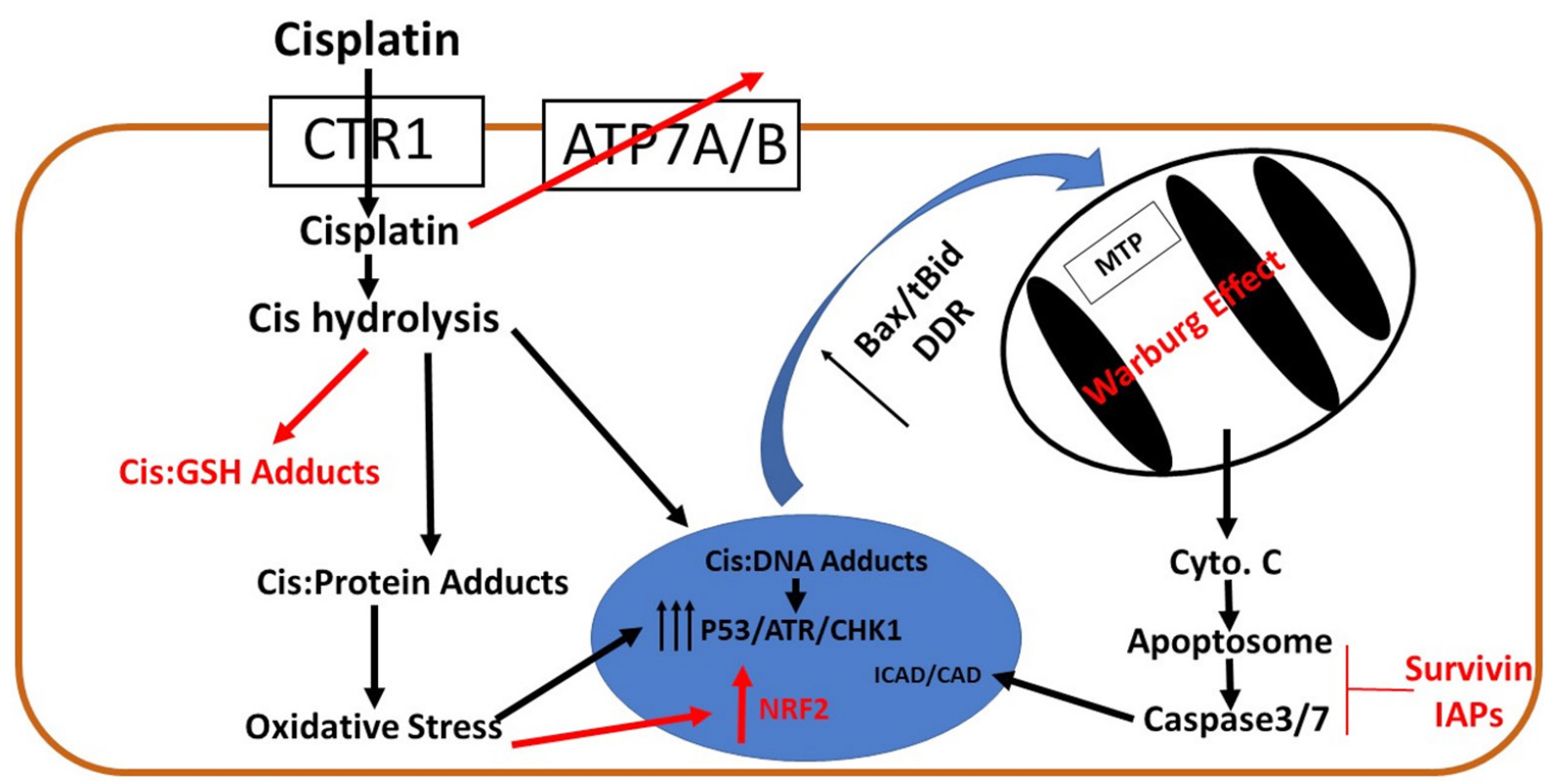

Figure 1. Mechanisms of cisplatin toxicity and resistance. Cisplatin is transported either actively by copper transporters such as CTR1 across the plasma membrane or passively through diffusion. Cisplatin can be actively transported out of the cell by metal transporters such as ATP7A and ATP7B and thus overexpression of these transporters is a mechanism of resistance. Cisplatin is hydrolyzed to its active form in the cytosol where it can bind proteins inducing oxidative stress or can be detoxified via cellular antioxidants like GSH. Active cisplatin binds purines on DNA which results in activation of p53 and the DNA damage response. P53 target genes like Bax and cellular stress from oxidative damage activate MOMP formation in the mitochondria, resulting in release of cytochrome $\mathrm{c}$ and activation of the apoptosome. Caspase3/7 cleave ICAD which release CAD and cleaves DNA leading to apoptosis. Black arrows notate events that promote toxicity, red arrows notate events that promote resistance. CTR1: copper transporter 1; Cis: cisplatin; GSH: glutathione; Bax: Bcl2 associated X-protein; tBid: truncated - BH3 domain interacting-domain death agonist; ATR: ataxia telengeicstasia and Rad3 related protein; CHK1: checkpoint kinase 1; Cyto C: cytochrome C; MTP: mitochondrial permeability transition pore; IAPs: inhibitors of apoptosis proteins; NRF2: nuclear factor erythroid-derived 2-like

in $\mathrm{BCa}$, interacts with cisplatin in $\mathrm{BCa}$, remains critical ${ }^{[23]}$. Finally, to execute cell death, mitochondrial stress results in release of components critical to the apoptosome including cytochrome $\mathrm{c}$ and apoptosis inducing factor (AIF) and this results in the activation of executor caspases (3/7) and apoptotic cell death through irreversible DNA cleavage ${ }^{[14]}$ [Figure 1]. Interplay between Bcl-2 family protein members such as Bid, Bax, and Bcl-XL as well as inhibitor of apoptosis (IAP) family members such as survivin further modulate the apoptotic response in cancer cells and are widely cited targets of natural products associated with chemotherapy.

While apoptosis is the most widely cited cause of cell death after cisplatin treatment, it should be noted that clinical specimens treated with cisplatin do not necessarily show pure apoptosis ${ }^{[24]}$. Furthermore, while in vitro experiments lead to activation of caspases, apoptosis may not be the predominant form of cell death at many doses of cisplatin in vitro ${ }^{[24,25]}$. Quantitative assessments of both necrosis and apoptosis found that in breast, gastric, and prostate cancers, levels of apoptosis induced by cisplatin were substantially less than levels of necrosis ${ }^{[24]}$. This may be due to inherent resistance to apoptosis in many cancer cells, and thus cell death input is eventually converted to cellular necrosis instead of cellular apoptosis as has been observed in other models ${ }^{[26]}$. As cellular necrosis is known to stimulate the immune environment via release of pro-inflammatory intracellular mediators, cisplatin-induced necrosis may benefit immunotherapy in the future by amplifying and sustaining an immune response ${ }^{[27,28]}$. Active trials combining immunotherapy and chemotherapy in $\mathrm{BCa}$ have thus far proved disappointing, but more trials are required to optimize this interaction ${ }^{[29]}$. It may be possible to stimulate an inflammatory environment while also killing tumor cells by initiating treatment with cisplatin and following up with sustained immunotherapy. 


\section{Mediators of cellular resistance to cisplatin}

Multiple mechanisms of resistance to cisplatin have been well established. These mechanisms follow the typical pattern of resistance mechanisms associated with alkylating agents such as cisplatin. This includes active transport into the cell, detoxification of cisplatin, altered DNA damage responses that prevent proapoptotic cellular signaling, direct inhibition of apoptosis, active export out of the cell, and inhibition of cellular energetics associated with Warburg metabolism (aerobic glycolysis). We will discuss these individual mechanisms and the mechanisms by which they reduce cisplatin efficacy.

Active transport of cisplatin

Cisplatin contains a central platinum that mediates a number of the biochemical properties of the compound. Transporters that control metal import and export from the cell, particularly copper transporters, also transport cisplatin and can result in resistance due to alterations in import and export of the drug ${ }^{[16]}$. These transporters are differentially regulated in many cancers where cisplatin is used clinically, and their regulation is associated with both resistance to therapy and off-target toxicity ${ }^{[30-32]}$. Moreover, laboratory models of ATP7A or ATP7B metal transporter overexpression result in resistance, consistent with the idea that active transport out of the cells can be just as important a mechanism as active transport into the cell ${ }^{[32]}$. These data coalesce around the idea that cisplatin transport is far from passive, and that uptake and export must be factored into both patient response and potential adverse events.

\section{Detoxification of cisplatin intracellularly}

Cisplatin resistance or susceptibility likely occurs to some degree in all cells, tumor or benign, at the level of detoxification. Increased levels of glutathione (GSH), activation of the transcription factor nuclear factor erythroid 2-related factor 2 (Nrf2), or indirect activation of Nrf2 via silencing of the Nrf2 binding partner Kelch Like ECH Associated Protein 1 (Keap1), upregulation of metal binding proteins such as metallothionein, and increased levels of thiol compounds can result in direct protection against cisplatin via detoxification ${ }^{[33-37]}$. All of these mechanisms are thought to work in part through direct detoxification of active cisplatin. GSH acts as a cellular sink for cisplatin and is easily replaced through synthesis of new GSH yielding continuous detoxification of cisplatin ${ }^{[3,35,36,38,39]}$. Depletion of GSH prior to cisplatin treatment with agents like buthionine sulfoximine dramatically enhances cisplatin efficacy; although as this would occur throughout the body, this mechanism is not a rational means for improving cisplatin clinically as it would also enhance cisplatin toxicity in susceptible tissues that were also depleted of $\mathrm{GSH}^{[38,39]}$. Metal binding proteins such as metallothionein are thought to directly bind the platinum in cisplatin via interactions with the many thiol groups present on cysteine rich metallothioneins, leading to direct detoxification. Blocking metallothionein expression would globally increase toxicity in all cells though, and thus is not a viable means for enhancing cisplatin. Notably, the ROS responsive transcription factor nuclear factor erythroid 2-related factor 2 ( Nrf2) can enhance synthesis of GSH through upregulation of GSH synthesizing proteins such as GCLC and GCLM ${ }^{[40]}$. Similarly, Nrf2 directly upregulates metal-binding proteins and phase I and phase II transporters that detoxify or otherwise prevent cisplatin toxicity ${ }^{[40]}$. This limits the amount of DNA/protein damage directly and leads to resistance. Many cancers are considered Nrf2 "addicted" and rely on Nrf2 activation ${ }^{[41]}$. A number of naturally derived compounds are putative Nrf2 activators and thus Nrf2 activation remains an understudied but likely mechanism through which many compounds can either block or promote cisplatin toxicity depending on their actions on $\mathrm{Nrf} 2$.

\section{Mutations in DNA damage repair}

A number of mutations and/or alterations in expression of genes associated with nucleotide excision repair (NER) can alter response to cisplatin. In BCa, recent research has focused on ERCC2 mutations as a major mediator of cisplatin sensitivity ${ }^{[42]}$. ERCC2 is normally responsible for NER of bulky adducts such as what is observed with cisplatin ${ }^{[43]}$. Mutation of ERCC2 confers sensitivity to cisplatin in vitro in cell lines and ERCC2 mutations are enriched significantly in responders to cisplatin ${ }^{[42,44]}$. Importantly, ablation of NER 
activity occurs with the majority of ERCC2 mutations, and experimentally initiated mutations had highly similar activities, consistent with the idea that most mutations of ERCC2 would convey susceptibility to cisplatin $^{[42,44]}$. These data concur with the major mechanism of cisplatin-mediated tissue injury being the formation of DNA adducts and the subsequent cellular response, as loss of NER would enhance cisplatinDNA adduct formation due to lack of repair, resulting in more potent cell death signaling. Monitoring $E R C C 2$ status remains a major potential biomarker of response to cisplatin and is a likely area of future clinical trials prospectively evaluating response to cisplatin based on ERCC2 status.

In contrast to ERCC2, ERCC1 positivity has highly variable results between studies. An initial study suggested low ERCC1 expression is associated with prolonged survival; however, a separate study two years in a larger cohort indicates patients that have no expression of ERCC1 have prolonged survival versus though patients that have some expression of ERCC $1^{[45-47]}$. It is possible that some degree of ERCC1 expression is required. Notably, it has been suggested this may not be related to cisplatin efficacy; although, other papers indicate that ERCC1 negative tumors benefit from neoadjuvant gemcitabine plus cisplatinbased chemotherapy ${ }^{[4-50]}$. Given the major discrepancies with ERCC1, it is unclear what data are correct; however, it may still be useful to distinguish what delineates the difference in these studies and determine more completely the role of ERCC1.

Mutations in base excision repair (BER) may also contribute to cisplatin effect. The BER protein Apurinic/ apyrimidinic endonuclease 1/redox factor-1 (APE1/Ref-1) has recently been identified as a target for enhancing the efficacy of cisplatin in bladder cancer ${ }^{[51]}$. APE-1 is upregulated in BCa which results in activation of transcription factors such as NF- $\kappa B$ that upregulates pro-survival proteins ${ }^{[52]}$. Inhibition of APE-1 with the novel compound E3330 increases the efficacy of cisplatin in addition to killing cells on its own ${ }^{[53]}$. Notably though, it remains unclear if this is specifically related to BCa, or the ability of E3330 to block BER, especially as APE1 may also regulate $\mathrm{Nrf}_{2}{ }^{[54]}$. Studies in breast cancer indicate E3330 enhances cisplatin resistance via blocking the pro-apoptotic response associated with DNA repair, and in contrast, suggests the lyase domain of polymerase $B$ is irrelevant, whereas knockdown of XRCC 1 results in sensitivity to cisplatin due to increased DNA damage ${ }^{[55]}$. Whether or not these mechanisms are universal between cancers thus remains in doubt; however, in $\mathrm{BCa}$, it appears that BER may be a target for improving cisplatin-based therapy. It needs to be determined if the actions are of APE- 1 and other BER proteins are directly through base excision repair, or instead through regulation of transcription factors such as Nrf2 that can also regulate the effects of cisplatin.

\section{Modulators of the Warburg effect}

Otto Warburg originally coined the idea that cancer cells primarily use glycolysis to produce ATP for energy ${ }^{[56]}$. Modern interpretation has emphatically demonstrated cancer cells have highly functional mitochondria and predominantly use oxidative respiration, and that while cancer cells upregulate many of the enzymes associated with glycolysis, the increased level of glucose uptake and glucose metabolism generate not only ATP but also oxidative biomass and metabolic intermediates which are equally if not more important ${ }^{[57,58]}$. A number of compounds that act on the Warburg Effect through inhibition of critical Warburg enzymes such as glucose transporter 1 (GLUT1), pyruvate dehydrogenase kinases (PDKs) or pyruvate kinase M2 (PKM2) also enhance cisplatin-based therapy, including in $\mathrm{BCa}^{[58-60]}$. Mechanisms delineated in other cancers indicate this is likely due to increased production of ROS by damaged mitochondria that are forced to use mitochondrial metabolism to generate the necessary biological intermediates ${ }^{[57,58,61]}$. Natural products targeting these enzymes have been discovered, and may serve as a source of chemotypes that can be further improved for targeting the Warburg Effect and overcoming cisplatin chemoresistance ${ }^{[57,62]}$. 


\section{Direct resistance to cisplatin induced apoptosis}

The primary mechanism noted in studies of cisplatin induced cell death is mitochondrially mediated caspase induced apoptosis ${ }^{[13,14]}$ [Figure 1]. Because apoptosis is a tightly controlled process mediated by both positive and negative regulators, cellular changes in the apoptotic process are not uncommon and can promote resistance to apoptosis ${ }^{[7,43]}$. $\mathrm{Bcl}-2$ family proteins such as survivin and $\mathrm{Bcl}$-xL promote resistance and these proteins are commonly upregulated in cancer ${ }^{[63]}$. In contrast, pro-apoptotic proteins such as Bax and Bid that initiate the mitochondrial permeability transition pore (MTP) necessary for release of proapoptotic factors from the mitochondria are commonly downregulated or dysregulated in cancer such that there is implicit resistance to apoptosis ${ }^{[14,63]}$. This may be a potential reason for the observation that, in spite of the focus on apoptosis as a major mediator of cisplatin toxicity, clinical cisplatin administration is consistent with mixed cell death (apoptosis and necrosis) ${ }^{[24]}$. A number of pathways that amplify the apoptotic signal, such as the c-Jun N-terminal kinase (JNK)-mediated pathway have been implicated in cisplatin toxicity as well and likely amplify upstream effects ${ }^{[64]}$. These pathways are confined to normal cells as JNK also amplifies cell death signals in tubular kidney cells exposed to cisplatin ${ }^{[65]}$. Natural products have routinely been shown to either amplify or inhibit associated pathways and thus may be useful for either treatment of cisplatin induced cytotoxicity or amplification of cisplatin induced anti-cancer activity.

\section{Cisplatin based chemotherapy in bladder cancer - predicting response in molecular subtypes}

While cisplatin remains standard of care only $30 \%-50 \%$ of patients respond and many have adverse events during the course of treatment ${ }^{[66,67]}$. Identification of biomarkers that can rapidly and effectively identify those patients that will respond is imperative. With the advent of molecular subtyping, a number of studies have demonstrated that BCa segregates into distinct groups based on genomic profiling ${ }^{[68-75]}$. Multiple groups have now established there is considerable difference between how these groups respond to cisplatin based, or other chemotherapy ${ }^{[7,76]}$. Tumors defined as luminal or basal subtype respond well to cisplatin; whereas recent information suggests neuroendocrine type or genomically neuroendocrine-like tumors and claudin-low tumors are poor responders to cisplatin with higher mortality rates ${ }^{[71,72,74,76,77]}$. Patients that respond well to cisplatin tend to have mutations in ERCC2 as mentioned above and high tumor mutational burdens ${ }^{[42,44,47]}$. Ultimately, these biomarkers are largely reflective of mechanisms that have been demonstrated in tumor cells and in human patients. Novel biomarkers that can improve which patients are designated for cisplatin-based chemotherapy are sorely needed. This would allow precision medicine initiatives aimed at reducing toxicity and improving therapy with all compounds, including natural products.

While this is not an exhaustive list of the mechanisms of cisplatin resistance, these mechanisms cover the majority of the known mediators. Cancer stemness and other similar factors are also highly associated with chemoresistance but, typically, stemness and other such forms of resistance directly involve those mechanisms listed above in some capacity. Understanding how current compounds affect these pathways as well as how to affect these pathways with novel compounds or synthetic derivatives remains critical to treating patients effectively with chemotherapy.

\section{NATURAL PRODUCTS - A VALIDATED SOURCE OF BIOLOGICALLY ACTIVE COMPOUNDS}

Natural products have previously produced agents that have been used in multiple ways to treat cancer, including as direct chemotherapeutics, chemo-sensitizing agents, and agents that prevent chemotoxicity ${ }^{[78-82]}$. Notably, mechanistic detail in this area has demonstrated that many of these compounds activate or repress some of the same pathways. At the same time, consensus mechanisms are commonly lacking. This is due to the fact that many of the compounds likely have multiple effects. As such, some compounds may promote apoptosis, but potentially through different mechanisms in different cell lines. Due to the sheer number of agents currently under investigation, we are going to focus on classes of drugs that could potentially synergize with cisplatin to overcome drug resistance and potential 
Table 1. Natural product derived inhibitors with potential synergy with cisplatin

\begin{tabular}{|c|c|c|c|}
\hline Class & Compounds & Source & Mode of action \\
\hline HSP90 inhibitors & $\begin{array}{l}\text { 17DMAG } \\
\text { 17AAG } \\
\text { Novobiocin }\end{array}$ & $\begin{array}{l}\text { Streptomyces } \\
\text { Species }\end{array}$ & $\begin{array}{l}\text { Inhibit AKT, B-Raf, etc... via direct downregulation of proteins due to lack } \\
\text { of chaperone mediated folding }\end{array}$ \\
\hline Flavone \& & Geninstein & Plant Derived & Induce apoptosis \\
\hline Isoflavones & Tangeretin & Citrus Peels & $\begin{array}{l}\text { Potentiate or synergize with cisplatin in } \mathrm{BCa} \\
\text { Genistein is a phytoestrogen and agonist of estrogen receptor }\end{array}$ \\
\hline Napthoquinones & $\begin{array}{l}\text { Shikonin } \\
\text { Alkannin }\end{array}$ & $\begin{array}{l}\text { Lithospermum } \\
\text { erythrorhizon }\end{array}$ & $\begin{array}{l}\text { Inhibition of PKM2 reverses Warburg Effect and produces antiproliferative } \\
\& \text { pro-apoptotic changes associated with pyruvate metabolism }\end{array}$ \\
\hline \multirow[t]{2}{*}{ Anthrocyclines } & $\begin{array}{l}\text { Adriamycin } \\
\text { Epirubicin }\end{array}$ & $\begin{array}{l}\text { Streptomyces } \\
\text { Species }\end{array}$ & Inhibits topoisomerase II resulting in catastrophic DNA damage \\
\hline & Evodiamine & Plant Derived & Evodiamine is a dual inhibitor of topoisomerase I and II \\
\hline Allyl disulfides & $\begin{array}{l}\text { (di \& tri) } \\
\text { allylsulfides }\end{array}$ & Garlic Derived & $\begin{array}{l}\text { Induce apoptosis, activates caspases, reduce phosphorylation of CHK } \\
\text { proteins, prevents resolution of double -strand breaks through an ATR/ } \\
\text { ATM dependent mechanism }\end{array}$ \\
\hline $\begin{array}{l}\text { Curcumin and } \\
\text { curcumin derivatives }\end{array}$ & Curcumin & $\begin{array}{l}\text { Plant (Turmeric) } \\
\text { Derived }\end{array}$ & $\begin{array}{l}\text { Induces apoptosis } \\
\text { Downregulating anti-apoptotic proteins such as } \mathrm{Bc} / 2 \text { \& survivin. } \\
\text { Upregulating Pro-apoptotic protein e.g., Bax. } \\
\text { Prevent upregulation of COX2 } \\
\text { Reduction in PGE2 }\end{array}$ \\
\hline Vitamin C & Ascorbic Acid & $\begin{array}{l}\text { Typically plant } \\
\text { derived }\end{array}$ & $\begin{array}{l}\text { ROS-induced cell death } \\
\text { May also downregulate HIFTA }\end{array}$ \\
\hline
\end{tabular}

Summary table of natural products, their source and mechanism of action. Numerous natural products have potential use in BCa. COX2: cyclooxygenase; PGE2: prostaglandin E2; CHK: checkpoint kinase; PKM2: pyruvate kinase M2; AKT: protein kinase B; B-Raf: serine/ threonine-protein kinase B-Raf; ATM: ataxia telangiectasia mutated; ATR: serine-threonine protein kinase ATR; ROS: reactive oxygen species; HIF1A: hypoxia inducible factor 1A

mechanisms, especially those with recently developed novel compounds. We have provided a Table [Table 1] to given a broad overview of the compound described in this section as well as their proposed methods of action and sources. This table should serve as an introductory guide to following compounds.

\section{HSP90 inhibitors}

Heat-shock protein 90 (HSP90) is a chaperone protein that stabilizes proteins necessary for tumor growth including protein kinase B (AKT), B-raf, and more ${ }^{[83]}$. HSP90A has routinely been demonstrated to be critical to cancer growth. Geldanamycin was the initial HSP90 inhibitor and was originally isolated from Streptomyces strains of bacteria and is thus a bacterially derived natural product. Natural product derived compounds such as geldanamycin and its analogues 17DMAG, 17AAG, and the more targeting HSB90 have proven successful in laboratory models but have limited potential clinically due to on-target toxicity associated with inhibition of all HSP90 isoforms ${ }^{[83,84]}$. Efforts aimed at specifically inhibiting HSP90 have yielded novel compounds that can inhibit BCa proliferation, but unfortunately led to the understanding that this induces a heat-shock response that is deleterious to the anti-cancer activity associated with HSP90 inhibition. In contrast, efforts to inhibit the C-terminus of HSP90, or efforts to inhibit the N-terminus of the $B$ form of HSP90 specifically have yielded inhibitors that may circumvent the heat shock response while also yielding efficacious therapeutics ${ }^{[84-86]}$. Novobiocin is a bacterially derived HSP90 inhibitor that targets the C-terminus of $\mathrm{HSP}_{90}{ }^{[87]}$. Novobiocin and derivatives do not initiate the heat shock response to the same degree and thus may be more viable means for inhibiting HSP90 long-term ${ }^{[84,87]}$. These compounds are derived from current HSP90 inhibitors and as such, the relative improvement seen with additional medicinal chemistry efforts may result in beneficial compounds. This is evidenced by prior studies indicating 17 -DMAG can effectively combat cisplatin resistance in $\mathrm{CD} 44^{+} \mathrm{BCa}$ causing stem-like cells ${ }^{[88]}$. The exact mechanism remains undetermined though, as HSP90 inhibitors can affect multiple pathways simultaneously given their broad range of protein targets. Further studies examining whether these compounds can functionally improve cisplatin-based therapy are warranted in HSP90 inhibitors with reduced toxicity. 


\section{Flavones and Isoflavones}

A number of flavones and isoflavones have anti-cancer activity. Many of these have direct anti-apoptotic activity through activation of caspases ${ }^{[89]}$. A few notable examples are listed here. Genistein is a wellknown plant-derived isoflavone and phytoestrogen that blocks BCa proliferation with multiple potentially relevant mechanisms ${ }^{[90]}$. Laboratory investigation of genistein indicates it is likely to induce apoptosis through actions on the intrinsic apoptotic pathway in addition to downregulation of nuclear factor nuclear factor kappa-light-chain-enhancer of activated $\mathrm{B}$ cells $(\mathrm{NF}-\kappa \mathrm{B})$ in $\mathrm{BCa}^{[90]}$. Mechanistic study on genistein indicates it may actually be significantly more complicated as it also is a full agonist of estrogen receptor $B$ and has significant estrogenic activity, i.e., it is a phytoestrogen ${ }^{[91]}$. Prior research with regards to estrogen receptors suggest there may be anti-apoptotic activity tied to estrogen receptor blockers in BCa that further complicates the mechanism ${ }^{[92]}$. In spite of these data, some epidemiological evidence points towards estrogen as potentially protective against $\mathrm{BCa}$, as women experience lower rates of $\mathrm{BCa}$ and some aspects of estrogen production are associated with reduced overall risk for $\mathrm{BCa}$ occurrence ${ }^{[93,94]}$. It may be that genistein, or genistein derived synthetic products, could be used to prevent cancer, especially in female patients that are post-menopausal. In order for this to happen, considerably more understanding of the role of estrogen and genistein in BCa would be needed.

Tangeretin is a flavone isolated from citrus peels with proven anti-cancer activity against BCa and also is synergizes with other established compounds such as adriamycin ${ }^{[95]}$. In spite of this, it is not well established how tangeretin induces apoptosis although some reports indicate mitochondrial dysfunction is critical to the apoptotic process similar to cisplatin ${ }^{[96]}$. Studies determining how tangeretin and other similar flavonoids with high abundance in citrus fruit interact with cisplatin and other critical chemotherapeutics are necessary to pinpoint dosing regimens and mechanisms. A number of other flavone or flavone derivatives have been put forth as potential chemotherapeutic agents; however, it should be noted that many of these compounds suffer from very poor pharmacology that ultimately prevents their usage in the clinic. As of yet there is no clinical evidence that flavones or isoflavones could potentiate or synergize with cisplatin in $\mathrm{BCa}$, although, given its success in other areas, this may be a fruitful area for combinatorial drug research that deserves further investigation.

\section{Napthoquinones}

Shikonin and its enantiomer alkannin are napthoquinones derived from Lithospermum erythrorhizon in addition to some Alkanna plant species with noted anti-cancer activity ${ }^{[59]}$. Shikonin has direct antiapoptotic activity in BCa cells and in xenograft tumor models and synergizes with cisplatin ${ }^{[59,62]}$. The mechanism appears to be through inhibition of $\mathrm{PKM} 22^{[59]}$. PKM2 inhibition reverses the Warburg Effect and thus produces anti-proliferative and pro-apoptotic changes by altering the production of both ATP and oxidative biomass associated with pyruvate metabolism ${ }^{[97]}$. Notably, this effect has been shown to be nonresponsive to the classical apoptosis inhibitor z-VAD-fmk and thus some doses of cisplatin may produce necroptosis rather than apoptosis ${ }^{[98]}$. Similar effects were found with other synthetic compounds acting on Warburg associated pathways in BCa indicating blockade of Warburg metabolism may be an effective means for treating BCa patients ${ }^{[58,60]}$. Importantly PKM2 is known to be upregulated in patients with BCa that are resistant to cisplatin, indicating this may be a common mechanism of resistance in patients ${ }^{[98]}$. This is an active area of novel research and both shikonin and other synthetic derivatives may be able to overcome chemoresistance ${ }^{[99,100]}$.

Anthracyclines: Anthracyclines are a class of drugs that inhibit topoisomerase II which results in cancer cell death due to catastrophic DNA damage. Possibly most notably is adriamycin, a component of one of the major chemotherapeutic regimens currently in use for BCa (dose dense MVAC). Adriamycin is directly derived from daunorubicin, a compound found in large quantities in Streptomyces bacterial strains. Adriamycin is thought to directly induce apoptosis in BCa cells through inhibition of topoisomerase II, 
eventually leading to cellular apoptosis due to uncontrolled DNA strand breakages. Adriamcyin improves cisplatin presumably through amplification of the apoptotic signal and enhanced DNA stress associated with DNA damage. Other anthracycline compounds such as epirubicin and 2"R)-4'-O-tetrahydropyranyldoxorubicin (THP) have extensively been investigated for the potential use as an instillation in superficial $\mathrm{BC}$ tumors ${ }^{[101,102]}$. Attempts at using intravesical epirubicin demonstrated that it did not improve BacillusCalmette-Guerin monotherapy ${ }^{[103]}$. Similarly, epirubicin alone fails to outperform BCG monotherapy on its own, although some effect with epirubicin alone was noted and the drug was well tolerated ${ }^{[104-106]}$. Given the prior success with adriamycin and known toxicity associated with topoisomerase II inhibitors future efforts should be aimed at developing inhibitors that are potent but with reduced toxicity. A number of new topoisomerase I and II inhibitors are currently being developed, some of which are derivatives of natural products $^{[107]}$. Evodiamine is a plant derived dual inhibitor of topoisomerase I and II, demonstrating efficacy against a number of tumor cell types including BCa; although notably, the proposed mechanism in BCa was attributed to inhibition of mTOR/S6K1 resulting in apoptosis ${ }^{[108,109]}$. In vitro IC50s for evodiamine are fairly high though, and thus improved compounds may also improve clinical merit. Molecular efforts aimed at understanding how to reduce toxicity, and synthetic efforts aimed at developing improved compounds with increased potency and reduced toxicity are necessary.

\section{Allyl disulfide and other garlic derivatives}

Compounds derived from garlic have potent anti-cancer activity, particularly diallyl disulfide, and triallyl disulfide. Early studies using non-orthotopically implanted murine tumors indicate large doses of garlic derivatives block cancer growth with dose limiting toxicity ${ }^{[110]}$. This activity is likely attributable to diallyl and triallyl disulfide as they directly induce apoptosis in BCa though activation of caspases ${ }^{[111,112]}$. In addition to apoptosis induction; allyl disulfides also reduce expression of metallomatrix proteinases which slows proliferation and invasion of BCa EJ cells which may contribute to their anti-tumorigenic effect ${ }^{[113]}$. Perhaps more importantly, diallyl sulfide induces phosphorylation of CHK proteins and potentially prevents resolution of double strand breaks through an ATR/ATM dependent mechanism ${ }^{[114,15]}$. As cisplatin also works through the formation of double strand breaks; and ERCC1 mutation is known to enhance cisplatin therapy, it is possible that synergy could be observed between diallyl/triallyl disulfide and cisplatin. Garlic derivatives and cisplatin were found to synergize in gastric cancer; although, this was postulated to function through attenuation of $\mathrm{Nrf2}$, a mechanism which has not been investigated in $\mathrm{BCa}$ with regards to garlic or garlic derivatives ${ }^{[116]}$. Even still, diallyl disulfide and other garlic derivatives may be highly efficacious, especially in cancers that are already known to be sensitive to cisplatin in human patients if the mechanism is ERCC1 dependent. Given the wide range of currently stated mechanisms, it is unclear what the exact mechanisms of diallyl disulfide induced apoptosis is, but it is consistently noted to block cancer proliferation induce apoptosis. Furthermore, relevant concentrations take extremely high doses that may not be reachable clinically in patients before the onset of toxicity.

\section{Curcumin and Curcumin derivatives}

Curcumin is a plant-based compound derived from turmeric and a potent inhibitor of BCa cancer growth. Curcumin induces apoptosis directly by downregulating anti-apoptotic proteins such as Bcl-2 and survivin, and upregulating pro-apoptotic proteins such as Bax ${ }^{[117]}$. Perhaps more importantly, curcumin inhibits the formation of superficial tumors in a rat model of orthotopic BCa carcinogenesis ${ }^{[17]}$. Similar to other compounds derived from natural products, mechanisms other than apoptosis have been suggested. Recent work in the BCa field indicates curcumin may block BCa progression through mechanisms not previously appreciated. Curcumin can prevent upregulation of cyclooxygenase-2 (COX-2) which results in reductions in prostaglandin E2 (PGE2) as COX-2 is the primary metabolizing gene for the production of $\mathrm{PGE}^{[118,119]}$. PGE2 levels promote tumor formation in $\mathrm{BCa}$ and other drugs targeting $\mathrm{PGE} 2$ production have demonstrated efficacy in $\mathrm{BCa}$, including potentiation or improvement of cisplatinbased therapy ${ }^{[8,120-123]}$. Importantly, whether this is directly due to inhibition of PGE2 production or due to 
other factors remains debatable, and thus, it is likely that if curcumin does inhibit PGE2 this is not the only mechanism through which it can block BCa proliferation. Blockade of PGE2 may be an important means through which curcumin could be used therapeutically in BCa as studies have demonstrated PGE2 is both involved in $\mathrm{BCa}$ tumor progression and cisplatin chemoresistance. Specifically, PGE2 levels contribute to a cancer stem cell like phenotype that is known to be resistant to cisplatin-based therapy though multiple mechanisms, as listed above ${ }^{[8]}$. Evidence indicates curcumin can directly prevent BCa proliferation in addition to the indirect evidence that curcumin-mediated blockade of PGE2 production would also have beneficial effects ${ }^{[8,124,125]}$. Curcumin improves both cisplatin based chemotherapy and gemcitabine based therapy as well ${ }^{[126,127]}$. Advancement of curcumin is unlikely as curcumin pharmacokinetics indicate it is rapidly metabolized when given orally and bioavailability is poor ${ }^{[128,129]}$. Novel curcumin derivatives with improved bioavailability are actively being developed, but must overcome major issues with bioavailability while retaining their anti-apoptotic effects if they are to be used successfully clinically. These studies may also be useful for determining the effects of PGE2 on BCa.

\section{Vitamin C}

A number of essential vitamins derived from different natural products have been investigated for anticancer activity. Oral doses of ascorbic acid (Vitamin C) do not produce systemic levels of Vitamin C consistent with anti-cancer activity due to active uptake by SLC23A1/SLC23A2 and high levels of excretion of non-absorbed Vitamin $C^{[130,131]}$. Vitamin $\mathrm{C}$ is known to kill cancer cells at levels obtained observed during intravenous delivery of vitamin $\mathrm{C}$, however ${ }^{[130-132]}$. The mechanism is thought to be through generation of hydrogen peroxide via an ascorbate radical formed during Vitamin $\mathrm{C}$ metabolism ${ }^{[133]}$. This radical is only minimally formed in blood which spares many blood cells normally damaged by chemotherapeutics. Alternate mechanisms including downregulation of hypoxia inducible factor $1 \alpha$ have also been proposed; although, how these mechanisms would directly kill cancer cells remains undetermined, whereas, increased levels of ROS are known to induce cell death ${ }^{[134]}$. Pharmacological levels of vitamin $\mathrm{C}$ are toxic to $\mathrm{BCa}$ cell lines which may occur in part through methylation of DNA and increased levels of 5-hydroxymethylcytosine which prevents malignancy in addition to established effects on ROS production $^{[135]}$. No study has directly tested synergy between cisplatin and Vitamin C as of yet; however, prior work indicates vitamin $\mathrm{C}$ can improve carboplatin-based therapy for ovarian cancer in laboratory models and was shown to prolong overall survival and time to disease recurrence in a small trial, although the prolonged survival did not meet statistical significance ${ }^{[132]}$. Clearly, given the solid safety profile of Vitamin C and its prior successes, it may be a viable means of improving cisplatin-based chemotherapy, or alternatively, improving therapy for patients that need less toxic chemotherapy regimens such as those that are ineligible for cisplatin.

It should be noted that many of the above chemicals are present in higher quantities in some specific diets designed to promote human health. One of the more common diets containing high quantities of plants and plant derivatives that includes isoflavones, garlic and garlic derivatives and other natural products is the Mediterranean Diet. This diet is associated with reduced risk of BCa in some analyses ${ }^{[136,137]}$. While oral consumption of these agents is likely to result in extensive metabolism and produce far lower systemic concentrations than direct administration, some benefit may be yielded from consistent intake of the diet. If this intake is related to natural product consumption and chemo-preventative effects, then understanding and isolating these specific agents or understanding their combined effects may be beneficial to treating BCa patients.

\section{Natural products and other forms of chemotherapy}

While cisplatin remains the mainstay chemotherapy for muscle invasive disease, it should be noted that immunotherapy in the form of BCG and immunotherapy in the form of checkpoint inhibitors are critical treatments for $\mathrm{BCa}$ in the modern era. Checkpoint inhibitors function through removing the blockade on $\mathrm{T}$ 
cells and re-establishing immunity; however, dysregulated inflammation is a known promoter of $\mathrm{BCa}^{[138,139]}$. As checkpoint inhibition as a therapeutic remains in its infancy, little information is available in this regard. Notably though, garlic derivatives are known to promote BCG effects and may improve the effects of other immunotherapy agents as well ${ }^{[140]}$. Similarly, reductions in PGE2 by curcumin may reduce the immunosuppressive environment and improve checkpoint inhibition. Given the diverse set of mechanisms associated with natural products, it will be imperative to define any potential benefit these agents may provide with immunotherapy.

\section{CONCLUSION}

Natural products have been a major source of chemotherapeutic drugs and will likely continue to be so. The number of therapeutics or chemotypes derived from natural products currently under study is substantial and far outweighs those discussed here. Both cisplatin and many of the discussed natural products have multiple mechanisms which may complicate our understanding of how to effectively dose these compounds and generate synergy with cisplatin. One thing missing from many of these agents are solid, high quality pharmacokinetic/pharmacodynamic studies that generate the data required for proper dosing of these compounds. These studies may further illuminate how we can alter structure-activity relationships of these compounds to improve their efficacy in vivo and duplicate the positive affects we have observed. The establishment of effective in vivo doses will then allow for more detailed mechanistic studies that target those concentrations that are achievable and effective in vivo. Increasing our understanding of how these compounds work is critical to developing synthetic derivatives that have improved pharmacokinetics and greater efficacy. Moreover, understanding the molecular basis of chemoresistance will allow us to position new drugs appropriately in those patients most likely to be responsive to chemotherapy. In summary, future studies aimed at refining current natural product derived compounds towards those that target specific aspects of cisplatin chemoresistance will be the most likely route to generate novel compounds that can improve patient chemotherapy.

\section{DECLARATIONS}

\section{Authors' contributions}

Writing, conception, figure generation: Rajendran G

Writing, editing: Taylor III JA

Writing, conception, figure generation, editing: Woolbright BL

\section{Availability of data and materials}

Not applicable.

\section{Financial support and sponsorship}

Research in this lab is funded by the Leo and Anne Albert Institute for Bladder Cancer Research and the Department of Defense DoD grant (CA190460). Woolbright BL is an American Urological Association Research Scholar and is funded by this award mechanism.

\section{Conflicts of interest}

All authors declared that there are no conflicts of interest.

\section{Ethical approval and consent to participate}

Not applicable.

\section{Consent for publication}

Not applicable. 


\section{Copyright}

(c) The Author(s) 2021.

\section{REFERENCES}

1. Kamat AM, Hahn NM, Efstathiou JA, et al. Bladder cancer. Lancet 2016;388:2796-810.

2. Penticuff JC, Woolbright BL, Sielecki TM, Weir SJ, Taylor JA 3rd. MIF family proteins in genitourinary cancer: tumorigenic roles and therapeutic potential. Nat Rev Urol 2019;16:318-28.

3. Grossman HB, Natale RB, Tangen CM, et al. Neoadjuvant chemotherapy plus cystectomy compared with cystectomy alone for locally advanced bladder cancer. $N$ Engl J Med 2003;349:859-66.

4. Scattoni V, Da Pozzo L, Nava L, et al. Five-year results of neoadjuvant cisplatin, methotrexate and vinblastine chemotherapy plus radical cystectomy in locally advanced bladder cancer. Eur Urol 1995;28:102-7.

5. Calabro F, Sternberg CN. Neoadjuvant and adjuvant chemotherapy in muscle-invasive bladder cancer. Eur Urol 2009;55:348-58.

6. Calabro F, Sternberg CN. Localized and locally advanced bladder cancer. Curr Treat Options Oncol 2002;3:413-28.

7. Anari F, O’Neill J, Choi W, et al. Neoadjuvant dose-dense gemcitabine and cisplatin in muscle-invasive bladder cancer: results of a phase 2 trial. Eur Urol Oncol 2018;1:54-60.

8. Kurtova AV, Xiao J, Mo Q, et al. Blocking PGE2-induced tumour repopulation abrogates bladder cancer chemoresistance. Nature 2015;517:209-13.

9. Pabla N, Dong Z. Cisplatin nephrotoxicity: mechanisms and renoprotective strategies. Kidney Int 2008;73:994-1007.

10. Miller RP, Tadagavadi RK, Ramesh G, Reeves WB. Mechanisms of cisplatin nephrotoxicity. Toxins (Basel) 2010;2:2490-518.

11. Ridzuan NRA, Rashid NA, Othman F, Budin SB, Hussan F, Teoh SL. Protective role of natural products in cisplatin-induced nephrotoxicity. Mini Rev Med Chem 2019;19:1134-43.

12. Demain AL, Vaishnav P. Natural products for cancer chemotherapy. Microb Biotechnol 2011;4:687-99.

13. Siddik ZH. Cisplatin: mode of cytotoxic action and molecular basis of resistance. Oncogene 2003;22:7265-79.

14. Galluzzi L, Senovilla L, Vitale I, et al. Molecular mechanisms of cisplatin resistance. Oncogene 2012;31:1869-83.

15. Ishida S, Lee J, Thiele DJ, Herskowitz I. Uptake of the anticancer drug cisplatin mediated by the copper transporter Ctr1 in yeast and mammals. Proc Natl Acad Sci U S A 2002;99:14298-302.

16. Song IS, Savaraj N, Siddik ZH, et al. Role of human copper transporter Ctr1 in the transport of platinum-based antitumor agents in cisplatin-sensitive and cisplatin-resistant cells. Mol Cancer Ther 2004;3:1543-9.

17. Eastman A. Glutathione-mediated activation of anticancer platinum(IV) complexes. Biochem Pharmacol 1987;36:4177-8.

18. Eastman A, Barry MA. Interaction of trans-diamminedichloroplatinum(II) with DNA: formation of monofunctional adducts and their reaction with glutathione. Biochemistry 1987;26:3303-7.

19. Eastman A. The formation, isolation and characterization of DNA adducts produced by anticancer platinum complexes. Pharmacol Ther 1987;34:155-66.

20. Woods D, Turchi JJ. Chemotherapy induced DNA damage response: convergence of drugs and pathways. Cancer Biol Ther 2013;14:379-89.

21. Sangster-Guity N, Conrad BH, Papadopoulos N, Bunz F. ATR mediates cisplatin resistance in a p 53 genotype-specific manner. Oncogene 2011;30:2526-33.

22. Konstantakou EG, Voutsinas GE, Karkoulis PK, Aravantinos G, Margaritis LH, Stravopodis DJ. Human bladder cancer cells undergo cisplatin-induced apoptosis that is associated with p53-dependent and p53-independent responses. Int J Oncol 2009;35:401-16.

23. Bunz F, Hwang PM, Torrance C, et al. Disruption of $\mathrm{p} 53$ in human cancer cells alters the responses to therapeutic agents. J Clin Invest 1999;104:263-9.

24. Kramer G, Erdal H, Mertens HJ, et al. Differentiation between cell death modes using measurements of different soluble forms of extracellular cytokeratin 18. Cancer Res 2004;64:1751-6.

25. Sancho-Martinez SM, Piedrafita FJ, Cannata-Andia JB, Lopez-Novoa JM, Lopez-Hernandez FJ. Necrotic concentrations of cisplatin activate the apoptotic machinery but inhibit effector caspases and interfere with the execution of apoptosis. Toxicol Sci 2011;122:73-85.

26. Jaeschke H, Lemasters JJ. Apoptosis versus oncotic necrosis in hepatic ischemia/reperfusion injury. Gastroenterology 2003;125:1246-57.

27. Hernandez C, Huebener P, Schwabe RF. Damage-associated molecular patterns in cancer: a double-edged sword. Oncogene 2016;35:5931-41.

28. Luedde T, Kaplowitz N, Schwabe RF. Cell death and cell death responses in liver disease: mechanisms and clinical relevance. Gastroenterology 2014;147:765-83.e4.

29. Galsky MD, Wang H, Hahn NM, et al. Phase 2 trial of gemcitabine, cisplatin, plus ipilimumab in patients with metastatic urothelial cancer and impact of DNA damage response gene mutations on outcomes. Eur Urol 2018;73:751-9.

30. Kilari D, Iczkowski KA, Pandya C, et al Copper transporter-CTR1 expression and pathological outcomes in platinum-treated muscleinvasive bladder cancer patients. Anticancer Res 2016;36:495-501.

31. Perde-Schrepler M, Fischer-Fodor E, Virag P, et al. The expression of copper transporters associated with the ototoxicity induced by platinum-based chemotherapeutic agents. Hear Res 2020;388:107893.

32. Katano K, Kondo A, Safaei R, et al. Acquisition of resistance to cisplatin is accompanied by changes in the cellular pharmacology of copper. Cancer Res 2002;62:6559-65.

33. Wang L, Wise JT, Zhang Z, Shi X. Progress and prospects of reactive oxygen species in metal carcinogenesis. Curr Pharmacol Rep 
2016;2:178-86.

34. Huang CF, Zhang L, Ma SR, et al. Clinical significance of Keap1 and Nrf2 in oral squamous cell carcinoma. PLoS One 2013;8:e83479.

35. Wang XJ, Sun Z, Villeneuve NF, et al. Nrf2 enhances resistance of cancer cells to chemotherapeutic drugs, the dark side of Nrf2. Carcinogenesis 2008;29:1235-43.

36. Godwin AK, Meister A, O’Dwyer PJ, Huang CS, Hamilton TC, Anderson ME. High resistance to cisplatin in human ovarian cancer cell lines is associated with marked increase of glutathione synthesis. Proc Natl Acad Sci U S A 1992;89:3070-4.

37. Bakka A, Endresen L, Johnsen AB, Edminson PD, Rugstad HE. Resistance against cis-dichlorodiammineplatinum in cultured cells with a high content of metallothionein. Toxicol Appl Pharmacol 1981;61:215-26.

38. Miyajima A, Nakashima J, Yoshioka K, Tachibana M, Tazaki H, Murai M. Role of reactive oxygen species in cisdichlorodiammineplatinum-induced cytotoxicity on bladder cancer cells. Br J Cancer 1997;76:206-10.

39. Miyajima A, Nakashima J, Tachibana M, Nakamura K, Hayakawa M, Murai M. N-acetylcysteine modifies cis-dichlorodiammineplatinuminduced effects in bladder cancer cells. Jpn J Cancer Res 1999;90:565-70.

40. Lee JS, Surh YJ. Nrf2 as a novel molecular target for chemoprevention. Cancer Lett 2005;224:171-84

41. Kitamura H, Motohashi H. NRF2 addiction in cancer cells. Cancer Sci 2018;109:900-11.

42. Van Allen EM, Mouw KW, Kim P, et al. Somatic ERCC2 mutations correlate with cisplatin sensitivity in muscle-invasive urothelial carcinoma. Cancer Discov 2014;4:1140-53.

43. Hanawalt PC, Spivak G. Transcription-coupled DNA repair: two decades of progress and surprises. Nat Rev Mol Cell Biol 2008;9:958-70.

44. Li Q, Damish AW, Frazier Z, et al. ERCC2 helicase domain mutations confer nucleotide excision repair deficiency and drive cisplatin sensitivity in muscle-invasive bladder cancer. Clin Cancer Res 2019;25:977-88.

45. Ozcan MF, Dizdar O, Dincer N, et al. Low ERCC1 expression is associated with prolonged survival in patients with bladder cancer receiving platinum-based neoadjuvant chemotherapy. Urol Oncol 2013;31:1709-15.

46. Mbeutcha A, Lucca I, Margulis V, et al. Prognostic role of ERCC1 protein expression in upper tract urothelial carcinoma following radical nephroureterectomy with curative intent. World J Urol 2016;34:1155-61.

47. Klatte T, Seitz C, Rink M, et al. ERCC1 as a prognostic and predictive biomarker for urothelial carcinoma of the bladder following radical cystectomy. J Urol 2015;194:1456-62.

48. Kawashima A, Takayama H, Tsujimura A. A review of ERCC1 gene in bladder cancer: implications for carcinogenesis and resistance to chemoradiotherapy. Adv Urol 2012;2012:812398.

49. Kawashima A, Nakayama M, Kakuta Y, et al. Excision repair cross-complementing group 1 may predict the efficacy of chemoradiation therapy for muscle-invasive bladder cancer. Clin Cancer Res 2011;17:2561-9.

50. Sun JM, Sung JY, Park SH, et al. ERCC1 as a biomarker for bladder cancer patients likely to benefit from adjuvant chemotherapy. $B M C$ Cancer 2012;12:187.

51. Kelley MR, Wikel JH, Guo C, et al. Identification and characterization of new chemical entities targeting apurinic/apyrimidinic endonuclease 1 for the prevention of chemotherapy-induced peripheral neuropathy. J Pharmacol Exp Ther 2016;359:300-9.

52. Shah F, Logsdon D, Messmann RA, Fehrenbacher JC, Fishel ML, Kelley MR. Exploiting the Ref-1-APE1 node in cancer signaling and other diseases: from bench to clinic. NPJ Precis Oncol 2017;1.

53. Fishel ML, Xia H, McGeown J, et al. Antitumor activity and mechanistic characterization of APE1/Ref-1 inhibitors in bladder cancer. Mol Cancer Ther 2019;18:1947-60.

54. Fishel ML, Wu X, Devlin CM, et al. Apurinic/apyrimidinic endonuclease/redox factor-1 (APE1/Ref-1) redox function negatively regulates NRF2. J Biol Chem 2015;290:3057-68.

55. Sawant A, Floyd AM, Dangeti M, Lei W, Sobol RW, Patrick SM. Differential role of base excision repair proteins in mediating cisplatin cytotoxicity. DNA Repair (Amst) 2017;51:46-59.

56. Warburg O. On the origin of cancer cells. Science 1956;123:309-14.

57. Vander Heiden MG, Cantley LC, Thompson CB. Understanding the Warburg effect: the metabolic requirements of cell proliferation. Science 2009;324:1029-33.

58. Woolbright BL, Rajendran G, Harris RA, Taylor JA 3rd. Metabolic flexibility in cancer: targeting the pyruvate dehydrogenase kinase:pyruvate dehydrogenase axis. Mol Cancer Ther 2019;18:1673-81.

59. Wang X, Zhang F, Wu XR. Inhibition of pyruvate kinase M2 markedly reduces chemoresistance of advanced bladder cancer to cisplatin. Sci Rep 2017;7:45983.

60. Woolbright BL, Choudhary D, Mikhalyuk A, et al. The role of pyruvate dehydrogenase kinase-4 (PDK4) in bladder cancer and chemoresistance. Mol Cancer Ther 2018;17:2004-12.

61. Bonnet S, Archer SL, Allalunis-Turner J, et al. A mitochondria-K+ channel axis is suppressed in cancer and its normalization promotes apoptosis and inhibits cancer growth. Cancer Cell 2007;11:37-51.

62. Liu W, Woolbright BL, Pirani K, et al. Tumor M2-PK: a novel urine marker of bladder cancer. PLoS One 2019;14:e218737.

63. Michaud WA, Nichols AC, Mroz EA, et al. Bcl-2 blocks cisplatin-induced apoptosis and predicts poor outcome following chemoradiation treatment in advanced oropharyngeal squamous cell carcinoma. Clin Cancer Res 2009;15:1645-54.

64. Yan D, An G, Kuo MT. C-Jun N-terminal kinase signalling pathway in response to cisplatin. J Cell Mol Med 2016;20:2013-9.

65. Oh CJ, Ha CM, Choi YK, et al. Pyruvate dehydrogenase kinase 4 deficiency attenuates cisplatin-induced acute kidney injury. Kidney Int 2017;91:880-95.

66. Zargar H, Zargar-Shoshtari K, Lotan Y, et al. Final pathological stage after neoadjuvant chemotherapy and radical cystectomy for bladder cancer-does pT0 predict better survival than pTa/Tis/T1? J Urol 2016;195:886-93. 
67. Zargar-Shoshtari K, Zargar H, Dinney CP, et al. Clinical and therapeutic factors associated with adverse pathological outcomes in clinically node-negative patients treated with neoadjuvant cisplatin-based chemotherapy and radical cystectomy. World $J$ Urol 2016;34:695-701.

68. Robertson AG, Kim J, Al-Ahmadie H, et al. Comprehensive molecular characterization of muscle-invasive bladder cancer. Cell 2017;171:540-56.e25.

69. McConkey DJ, Choi W, Shen Y, et al. A prognostic gene expression signature in the molecular classification of chemotherapy-naive urothelial cancer is predictive of clinical outcomes from neoadjuvant chemotherapy: a phase 2 trial of dose-dense methotrexate, vinblastine, doxorubicin, and cisplatin with bevacizumab in urothelial cancer. Eur Urol 2016;69:855-62.

70. McConkey DJ, Choi W, Dinney CP. Genetic subtypes of invasive bladder cancer. Curr Opin Urol 2015;25:449-58.

71. Choi W, Czerniak B, Ochoa A, et al. Intrinsic basal and luminal subtypes of muscle-invasive bladder cancer. Nat Rev Urol 2014;11:400-10.

72. Dyrskjot L. Molecular subtypes of bladder cancer: academic exercise or clinical relevance? Eur Urol 2019;75:433-4.

73. Thomsen MBH, Nordentoft I, Lamy P, et al. Comprehensive multiregional analysis of molecular heterogeneity in bladder cancer. Sci Rep 2017;7:11702.

74. Hedegaard J, Lamy P, Nordentoft I, et al. Comprehensive transcriptional analysis of early-stage urothelial carcinoma. Cancer Cell 2016;30:27-42.

75. Sjodahl G, Eriksson P, Liedberg F, Hoglund M. Molecular classification of urothelial carcinoma: global mRNA classification versus tumour-cell phenotype classification. J Pathol 2017;242:113-25.

76. Grivas P, Bismar TA, Alva AS, et al. Validation of a neuroendocrine-like classifier confirms poor outcomes in patients with bladder cancer treated with cisplatin-based neoadjuvant chemotherapy. Urol Oncol 2020;38:262-8.

77. Batista da Costa J, Gibb EA, Bivalacqua TJ, et al. Molecular characterization of neuroendocrine-like bladder cancer. Clin Cancer Res 2019;25:3908-20.

78. de Oliveira Junior RG, Christiane Adrielly AF, da Silva Almeida JRG, Grougnet R, Thiery V, Picot L. Sensitization of tumor cells to chemotherapy by natural products: a systematic review of preclinical data and molecular mechanisms. Fitoterapia 2018;129:383-400.

79. Kotecha R, Takami A, Espinoza JL. Dietary phytochemicals and cancer chemoprevention: a review of the clinical evidence. Oncotarget 2016;7:52517-29.

80. Singh S, Sharma B, Kanwar SS, Kumar A. Lead phytochemicals for anticancer drug development. Front Plant Sci 2016;7:1667.

81. Vinod BS, Maliekal TT, Anto RJ. Phytochemicals as chemosensitizers: from molecular mechanism to clinical significance. Antioxid Redox Signal 2013;18:1307-48.

82. Wang H, Khor TO, Shu L, et al. Plants vs. cancer: a review on natural phytochemicals in preventing and treating cancers and their druggability. Anticancer Agents Med Chem 2012;12:1281-305.

83. Schopf FH, Biebl MM, Buchner J. The HSP90 chaperone machinery. Nat Rev Mol Cell Biol 2017;18:345-60.

84. Khandelwal A, Kent CN, Balch M, et al. Structure-guided design of an Hsp90beta N-terminal isoform-selective inhibitor. Nat Commun 2018;9:425.

85. Garg G, Forsberg LK, Zhao H, Blagg BSJ. Development of phenyl cyclohexylcarboxamides as a novel class of Hsp90 C-terminal inhibitors. Chemistry 2017;23:16574-85.

86. Whitesell L, Bagatell R, Falsey R. The stress response: implications for the clinical development of hsp90 inhibitors. Curr Cancer Drug Targets 2003;3:349-58.

87. Marcu MG, Chadli A, Bouhouche I, Catelli M, Neckers LM. The heat shock protein 90 antagonist novobiocin interacts with a previously unrecognized ATP-binding domain in the carboxyl terminus of the chaperone. J Biol Chem 2000;275:37181-6.

88. Tatokoro M, Koga F, Yoshida S, et al. Potential role of Hsp90 inhibitors in overcoming cisplatin resistance of bladder cancer-initiating cells. Int J Cancer 2012;131:987-96.

89. Abotaleb M, Samuel SM, Varghese E, et al. Flavonoids in cancer and apoptosis. Cancers (Basel) 2018;11.

90. Singh AV, Franke AA, Blackburn GL, Zhou JR. Soy phytochemicals prevent orthotopic growth and metastasis of bladder cancer in mice by alterations of cancer cell proliferation and apoptosis and tumor angiogenesis. Cancer Res 2006;66:1851-8.

91. Kuiper GG, Lemmen JG, Carlsson B, et al. Interaction of estrogenic chemicals and phytoestrogens with estrogen receptor beta. Endocrinology 1998;139:4252-63.

92. Hoffman KL, Lerner SP, Smith CL. Raloxifene inhibits growth of RT4 urothelial carcinoma cells via estrogen receptor-dependent induction of apoptosis and inhibition of proliferation. Horm Cancer 2013;4:24-35.

93. McGrath M, Michaud DS, De Vivo I. Hormonal and reproductive factors and the risk of bladder cancer in women. Am J Epidemiol 2006;163:236-44.

94. Daugherty SE, Lacey JV Jr, Pfeiffer RM, Park Y, Hoover RN, Silverman DT. Reproductive factors and menopausal hormone therapy and bladder cancer risk in the NIH-AARP Diet and Health Study. Int J Cancer 2013;133:462-72.

95. Lin JJ, Huang CC, Su YL, et al. Proteomics analysis of tangeretin-induced apoptosis through mitochondrial dysfunction in bladder cancer cells. Int J Mol Sci 2019;20.

96. Dong Y, Cao A, Shi J, et al. Tangeretin, a citrus polymethoxyflavonoid, induces apoptosis of human gastric cancer AGS cells through extrinsic and intrinsic signaling pathways. Oncol Rep 2014;31:1788-94.

97. Liu VM, Vander Heiden MG. The role of pyruvate kinase M2 in cancer metabolism. Brain Pathol 2015;25:781-3.

98. Wang Y, Hao F, Nan Y, et al. PKM2 inhibitor shikonin overcomes the cisplatin resistance in bladder cancer by inducing necroptosis. Int $J$ Biol Sci 2018;14:1883-91.

99. Ning X, Qi H, Li R, et al. Discovery of novel naphthoquinone derivatives as inhibitors of the tumor cell specific M2 isoform of pyruvate 
kinase. Eur J Med Chem 2017;138:343-52.

100. Vander Heiden MG, Christofk HR, Schuman E, et al. Identification of small molecule inhibitors of pyruvate kinase M2. Biochem Pharmacol 2010;79:1118-24.

101. Lamm DL, McGee WR, Hale K. Bladder cancer: current optimal intravesical treatment. Urol Nurs 2005;25:323-6, 31-2.

102. Onrust SV, Wiseman LR, Goa KL. Epirubicin: a review of its intravesical use in superficial bladder cancer. Drugs Aging 1999;15:307-33.

103. Tozawa K, Okamura T, Yamada Y, Hayashi Y, Sasaki S, Kohri K. Prevention of the recurrence of superficial bladder cancers: intravesical instillation of bacillus calmette-guerin versus bacillus calmette-guerin plus epirubicin. Asian Pac J Cancer Prev 2000;1:217-20.

104. Melekos MD, Chionis HS, Paranychianakis GS, Dauaher HH. Intravesical 4'-epi-doxorubicin (epirubicin) versus bacillus CalmetteGuerin. A controlled prospective study on the prophylaxis of superficial bladder cancer. Cancer 1993;72:1749-55.

105. Melekos MD, Chionis H, Pantazakos A, Fokaefs E, Paranychianakis G, Dauaher H. Intravesical bacillus Calmette-Guerin immunoprophylaxis of superficial bladder cancer: results of a controlled prospective trial with modified treatment schedule. J Urol 1993;149:744-8.

106. Melekos MD, Dauaher H, Fokaefs E, Barbalias G. Intravesical instillations of 4-epi-doxorubicin (epirubicin) in the prophylactic treatment of superficial bladder cancer: results of a controlled prospective study. J Urol 1992;147:371-5.

107. Hevener K, Verstak TA, Lutat KE, Riggsbee DL, Mooney JW. Recent developments in topoisomerase-targeted cancer chemotherapy. Acta Pharm Sin B 2018;8:844-61.

108. Pan X, Hartley JM, Hartley JA, White KN, Wang Z, Bligh SW. Evodiamine, a dual catalytic inhibitor of type I and II topoisomerases, exhibits enhanced inhibition against camptothecin resistant cells. Phytomedicine 2012;19:618-24.

109. Zhang T, Qu S, Shi Q, He D, Jin X. Evodiamine induces apoptosis and enhances TRAIL-induced apoptosis in human bladder cancer cells through mTOR/S6K1-mediated downregulation of Mcl-1. Int J Mol Sci 2014;15:3154-71.

110. Riggs DR, DeHaven JI, Lamm DL. Allium sativum (garlic) treatment for murine transitional cell carcinoma. Cancer 1997;79:1987-94.

111. Wang YB, Qin J, Zheng XY, Bai Y, Yang K, Xie LP. Diallyl trisulfide induces Bcl-2 and caspase-3-dependent apoptosis via downregulation of Akt phosphorylation in human T24 bladder cancer cells. Phytomedicine 2010;17:363-8.

112. Kim WT, Seo SP, Byun YJ, et al. Garlic extract in bladder cancer prevention: evidence from T24 bladder cancer cell xenograft model, tissue microarray, and gene network analysis. Int J Oncol 2017;51:204-12.

113. Shin DY, Cha HJ, Kim GY, Kim WJ, Choi YH. Inhibiting invasion into human bladder carcinoma 5637 cells with diallyl trisulfide by inhibiting matrix metalloproteinase activities and tightening tight junctions. Int J Mol Sci 2013;14:19911-22.

114. Kuo CH, Leu YL, Wang TH, et al. A novel DNA repair inhibitor, diallyl disulfide (DADS), impairs DNA resection during DNA doublestrand break repair by reducing Sae2 and Exo1 levels. DNA Repair (Amst) 2019;82:102690.

115. Ling H, Lu LF, He J, Xiao GH, Jiang H, Su Q. Diallyl disulfide selectively causes checkpoint kinase-1 mediated G2/M arrest in human MGC803 gastric cancer cell line. Oncol Rep 2014;32:2274-82.

116. Jiang XY, Zhu XS, Xu HY, et al. Diallyl trisulfide suppresses tumor growth through the attenuation of Nrf2/Akt and activation of p38/ JNK and potentiates cisplatin efficacy in gastric cancer treatment. Acta Pharmacol Sin 2017;38:1048-58.

117. Tian B, Wang Z, Zhao Y, et al. Effects of curcumin on bladder cancer cells and development of urothelial tumors in a rat bladder carcinogenesis model. Cancer Lett 2008;264:299-308.

118. Sharma RA, Euden SA, Platton SL, et al. Phase I clinical trial of oral curcumin: biomarkers of systemic activity and compliance. Clin Cancer Res 2004;10:6847-54.

119. Plummer SM, Holloway KA, Manson MM, et al. Inhibition of cyclo-oxygenase 2 expression in colon cells by the chemopreventive agent curcumin involves inhibition of NF-kappaB activation via the NIK/IKK signalling complex. Oncogene 1999;18:6013-20.

120. Mohammed SI, Dhawan D, Abraham S, et al. Cyclooxygenase inhibitors in urinary bladder cancer: in vitro and in vivo effects. Mol Cancer Ther 2006;5:329-36.

121. Mizutani Y, Nakanishi H, Li YN, Sato N, Kawauchi A, Miki T. Enhanced sensitivity of bladder cancer cells to cisplatin mediated cytotoxicity and apoptosis in vitro and in vivo by the selective cyclooxygenase-2 inhibitor JTE-522. J Urol 2004;172:1474-9.

122. Mohseni H, Zaslau S, McFadden D, Riggs DR, Jackson BJ, Kandzari S. COX-2 inhibition demonstrates potent anti-proliferative effects on bladder cancer in vitro. $J$ Surg Res 2004;119:138-42.

123. Woolbright BL, Pilbeam CC, Taylor JA 3rd. Prostaglandin E2 as a therapeutic target in bladder cancer: From basic science to clinical trials. Prostaglandins Other Lipid Mediat 2020;148:106409.

124. Liang Z, Lu L, Mao J, Li X, Qian H, Xu W. Curcumin reversed chronic tobacco smoke exposure induced urocystic EMT and acquisition of cancer stem cells properties via Wnt/beta-catenin. Cell Death Dis 2017;8:e3066.

125. Li Y, Lin K, Yang Z, et al. Bladder cancer stem cells: clonal origin and therapeutic perspectives. Oncotarget 2017;8:66668-79.

126. Kamat AM, Sethi G, Aggarwal BB. Curcumin potentiates the apoptotic effects of chemotherapeutic agents and cytokines through downregulation of nuclear factor-kappaB and nuclear factor-kappaB-regulated gene products in IFN-alpha-sensitive and IFN-alpha-resistant human bladder cancer cells. Mol Cancer Ther 2007;6:1022-30.

127. Park BH, Lim JE, Jeon HG, et al. Curcumin potentiates antitumor activity of cisplatin in bladder cancer cell lines via ROS-mediated activation of ERK1/2. Oncotarget 2016;7:63870-86.

128. Sharma RA, McLelland HR, Hill KA, et al. Pharmacodynamic and pharmacokinetic study of oral Curcuma extract in patients with colorectal cancer. Clin Cancer Res 2001;7:1894-900.

129. Ireson C, Orr S, Jones DJ, et al. Characterization of metabolites of the chemopreventive agent curcumin in human and rat hepatocytes and in the rat in vivo, and evaluation of their ability to inhibit phorbol ester-induced prostaglandin E2 production. Cancer Res 2001;61:1058-64.

130. Hoffer LJ, Levine M, Assouline S, et al. Phase I clinical trial of i.v. ascorbic acid in advanced malignancy. Ann Oncol 2008;19:1969-74. 
131. Padayatty SJ, Sun H, Wang Y, et al. Vitamin C pharmacokinetics: implications for oral and intravenous use. Ann Intern Med 2004;140:533-7.

132. Ma Y, Chapman J, Levine M, Polireddy K, Drisko J, Chen Q. High-dose parenteral ascorbate enhanced chemosensitivity of ovarian cancer and reduced toxicity of chemotherapy. Sci Transl Med 2014;6:222ra18.

133. Parrow NL, Leshin JA, Levine M. Parenteral ascorbate as a cancer therapeutic: a reassessment based on pharmacokinetics. Antioxid Redox Signal 2013;19:2141-56.

134. Campbell EJ, Vissers MC, Bozonet S, Dyer A, Robinson BA, Dachs GU. Restoring physiological levels of ascorbate slows tumor growth and moderates HIF-1 pathway activity in Gulo(-/-) mice. Cancer Med 2015;4:303-14.

135. Peng D, Ge G, Gong Y, et al. Vitamin C increases 5-hydroxymethylcytosine level and inhibits the growth of bladder cancer. Clin Epigenetics 2018;10:94.

136. Witlox WJA, van Osch FHM, Brinkman M, et al. An inverse association between the Mediterranean diet and bladder cancer risk: a pooled analysis of 13 cohort studies. Eur J Nutr 2020;59:287-96.

137. Buckland G, Ros MM, Roswall N, et al. Adherence to the Mediterranean diet and risk of bladder cancer in the EPIC cohort study. Int $J$ Cancer 2014;134:2504-11.

138. Ferro M, De Cobelli O, Buonerba C, et al. Modified Glasgow Prognostic Score Is Associated With Risk Of Recurrence In Bladder Cancer Patients After Radical Cystectomy: a Multicenter Experience. Medicine (Baltimore) 2015;94:e1861.

139. Sui X, Lei L, Chen L, Xie T, Li X. Inflammatory microenvironment in the initiation and progression of bladder cancer. Oncotarget 2017;8:93279-94.

140. Lamm DL, Riggs DR. Enhanced immunocompetence by garlic: role in bladder cancer and other malignancies. J Nutr 2001;131:1067S-70. 